memory B-cells and no correlation between PDAI and ICSA antibody levels.

Discussion: Both dsg antibody levels and B-cell counts can predict relapse in subgroups of rituximab-treated refractory PV patients.

\section{SOLUBLE FACTORS FROM PACKED RED BLOOD CELLS AUGMENTED LPS INDUCED MONOCYTE PRODUCTION OF IL-1B AND CASPASE-1}

Robert Flower $^{1}$, Fenny Chong ${ }^{1}$, Kelly Rooks ${ }^{1}$, Melinda Dean ${ }^{1,2}$ ${ }^{1}$ Research and Development, Australian Red Cross Lifeblood, Kelvin Grove, Qld, Australia; and ${ }^{2}$ School of Health and Sport Sciences, University of the Sunshine Coast, Moreton Bay, Qld, Australia

Introduction: During routine storage packed red blood cells (PRBC) undergo biochemical and morphological changes, and soluble mediators accumulate which have been hypothesised to contribute to poor outcomes post-transfusion. Interleukin (IL)-1 $\beta$ is a pro-inflammatory cytokine critical for cell proliferation and differentiation. We investigated whether PRBC transfusion modulated IL-1 $\beta$ driven inflammation and assessed whether macrophage inhibitory factor (MIF) was involved.

Methods: Isolated monocytes were co-incubated with PRBC supernatants (collected at D2, D14, D28, D42) +/- lipopolysaccharide (LPS, $1 \mu \mathrm{g} / \mathrm{mL}$ ) or LPS+ATP (positive control) for 4 hours, $37^{\circ} \mathrm{C}, 5 \% \mathrm{CO}_{2}$. IL- $1 \beta$ and caspase- 1 were quantified in PRBC-SN and culture SN. MIF was quantified in PRBC-SN and then recombinant MIF (rMIF) +/- LPS added to model 2-3 unit of transfusion. Results were analysed by ANOVA (compared to untreated or LPS alone, $p<0.05$ ).

Results: PRBC-SN increased LPS-induced monocyte production of IL-1 $\beta(p<0.05)$ and caspase-1 $(p<0.05)$. MIF was present in D2 (19 ng/mL) and D42 (247 ng/mL) PRBC-SN. rMIF did not modulate LPS-induced IL-1 $\beta$ or caspase-1 production from monocytes.

Conclusions: Soluble factors in PRBC augmented LPS-induced IL-1 $\beta$ and caspase-1 production in monocytes suggesting inflammasome activation. There was no evidence that MIF was responsible for modulating IL-1 $\beta$ production and further investigation into the other immunomodulatory mediators from PRBC is warranted.

\section{SEQUENTIAL INVOLVEMENT OF URETERS AND KIDNEYS WITH IGG4 DISEASE LEADING TO NEPHRECTOMY: A CASE REPORT}

\author{
Hannah $\mathrm{Hu}^{1}$, Arthur Vasilaras ${ }^{2}$, Geoffrey Watson ${ }^{3,4}$, \\ Amruta Trivedi ${ }^{1}$, Frederick Lee ${ }^{1,4}$ \\ ${ }^{1}$ Department of Clinical Immunology and Allergy, Royal Prince \\ Alfred Hospital, Sydney, Australia; ${ }^{2}$ Department of Urology, \\ Royal Prince Alfred Hospital, Sydney, Australia; ${ }^{3}$ Department \\ of Tissue Pathology, Royal Prince Alfred Hospital, Sydney, \\ Australia; and ${ }^{4}$ University of Sydney, Sydney, Australia
}

We present a 63-year-old male with IgG4-related retroperitoneal fibrosis, causing secondary obstructive pyelonephritis. Imaging of the retroperitoneum was not informative, and prior to the diagnosis being made a left, simple nephrectomy was performed for the obstructive uropathy.
The diagnosis was later suspected because of progression to contralateral obstructive uropathy with subtle medial displacement of the ureter. It was established based on serum IgG subsets, retrospective immunostaining of the nephrectomy tissues, and supported by FDG-PET showing low-level uptake in the retroperitoneum.

This case highlights the importance of awareness of $\mathrm{IgG} 4$ disease in the context of obstructive uropathy, and the need for clinical/ imaging correlation when histologically assessing a nephrectomy. The histological pattern of established ureteropelvic mural fibrosis depositing circumferentially, associated with ongoing mural inflammation including plasma cells, but sparing the urothelium as well as showing a relative paucity of fibroblasts and histiocytes may be clues to the diagnosis.

The management of IgG4-related disease is immunosuppression (trying to stall its progression), as well as managing the complications caused by the already-deposited fibrosis.

IgG4 retroperitoneal disease is often a multidisciplinary diagnosis, requiring a high level of suspicion and the assessment of multiple diagnostic parameters. Diagnosis at its earlier stages is challenging.

\section{A FIVE-YEAR RETROSPECTIVE AUDIT OF ANTI- MYELOPEROXIDASE ANTIBODY TITRES MEASURED USING A COMMERCIAL CHEMILUMINESCENT IMMUNOASSAY}

\author{
Celina Jin ${ }^{1,2}$, Euan McNaughton ${ }^{1}$, Candice $\mathrm{Li}^{1}$, \\ Carolyn Hawkins ${ }^{1,2}$ \\ ${ }^{1}$ Department of Immunopathology, ACT Pathology, Canberra \\ ACT, Australia; and ${ }^{2}$ Department of Immunology, The \\ Canberra Hospital, Canberra ACT, Australia
}

Background: In 2015, ACT Pathology transitioned to a commercial chemiluminescent immunoassay (QUANTA-Flash MPO, Inova Diagnostics) to measure anti-MPO IgG antibodies. Monitoring of internal performance check sera identified discordant results following a change in reagent lot. To investigate this further, we conducted a five-year retrospective audit of all anti-MPO titres measured using this assay.

Methods: Samples were stratified according to titre (negative $<6$ $\mathrm{IU} / \mathrm{mL}$, low-positive $6-23 \mathrm{IU} / \mathrm{mL}$ and high-positive $\geq 24 \mathrm{IU} /$ $\mathrm{mL}$ ), clinical information provided on request forms, ANCA pattern as detected by indirect immunofluorescence (IIF), and reagent lot number.

Results: A total of 2005 anti-MPO titres were measured between 15 May 2015 and 13 August 2019. The mean number of tests performed each month doubled between 2015-2016 (25 tests/month) to 2017-2019 (50 tests/month). This was associated with increased testing of patients with known AAV. Some variation in the percentage of low-positive $(5.8-16.4 \%)$ and high-positive $(7.7-21.4 \%)$ results was observed between the 12 reagent lots used, however this may have been confounded by duration of use (range 2-296 days). The predicted probability of p-ANCA detection was significantly higher in patients with higher anti-MPO IgG titres $(\mathrm{OR}=17.66,95 \%$ CI 13.72-21.13, $p<0.0001$ ).

Conclusion: Anti-MPO IgG testing at ACT Pathology has increased over the last five years. While some variation between reagent lots has been observed, this has not significantly affected overall assay performance. 